Cahiers d'études italiennes

$7 \mid 2008$

NOVECENTO... E DINTORNI

Images littéraires de la société contemporaine (3)

\title{
Le portrait et la catastrophe
}

\section{Claude Ambroise}

\section{OpenEdition}

\section{Journals}

Édition électronique

URL : http://journals.openedition.org/cei/946

DOI : $10.4000 /$ cei.946

ISSN : 2260-779X

Éditeur

UGA Éditions/Université Grenoble Alpes

\section{Édition imprimée}

Date de publication : 15 mai 2008

Pagination : 385-395

ISBN : 978-2-84310-121-2

ISSN : $1770-9571$

Référence électronique

Claude Ambroise, «Le portrait et la catastrophe », Cahiers d'études italiennes [En ligne], 7| 2008, mis en ligne le 15 novembre 2009, consulté le 27 avril 2019. URL : http://journals.openedition.org/cei/946 ; DOI : 10.4000/cei.946 


\title{
LE PORTRAIT ET LA CATASTROPHE
}

\author{
Claude Ambroise \\ Université Stendhal-Grenoble 3
}

La différence, j'ai voulu la repérer entre les lignes et dans les marges de $I l$ sorriso dell'ignoto marinaio, un texte publié en 1976, qui n'est pas le premier écrit de Consolo, mais qui est, à ses yeux, et au regard de la critique également, un livre fondateur ${ }^{1 *}$. Pour préciser cette dernière notion à l'aide d'un exemple géographiquement voisin, on rappellera que Le parrocchie di Regalpetra (1956) de Sciascia n'est pas le premier texte de cet auteur, mais que celui-ci y voyait le texte fondateur de son oeuvre.

Consolo est revenu sur cette fonction du Sorriso dell'ignoto marinaio, dans un court écrit de 1997, qui aujourd'hui figure dans Di qua dal faro (1999), ainsi qu'en appendice au roman lui-même dans l'actuelle édition Oscar Mondadori, dont je tire les citations qui vont suivre.

Précisant qu'il a vécu dans les pays des Nebrodi, Consolo remarque qu'on n'y note que de rares révoltes populaires «come quella rirsorgimentale di Alcàra Li Fusi, tramandata più dal racconto orale che dalla storiografia». Je souligne l'opposition entre la tradition orale et l'historiographie.

À propos du tableau d'Antonello, il indique que celui-ci, quand il pensait le livre à faire, devait être, en quelque sorte, la pointe névralgique d'une «invenzione, costruzione, soprattutto linguistica».

Troisième et dernière citation, un peu longue, toujours tirée de cette revisitation consolienne, et vraiment en harmonie avec l'objet d'un colloque sur la différence:

La struttura poi del romanzo, la cui organicità è spezzata, intervallata da inserti documentari o da allusive, ironiche citazioni, lo connotava come metaromanzo o antiromanzo storico. [...] per me il suo linguaggio e la sua struttura volevano indicare il superamento, in senso etico, estetico, attraverso mimesi, parodia, fratture, spezzature, oltranze immaginarie, dei romanzi d'intreccio dispiegati e dominati dall'autore, di tutti i linguaggi logici, illuministici, che, nella loro limpida, serena geometrizzazione, escludevano le "voci" dei margini.

* Notes p. 395. 
Je ne désire pas proposer, directement du moins, une interprétation idéologique du roman de 1976, en relation avec l'analyse qu'on peut produire du moment historique où il est écrit, ni non plus avec les prises de position politiques de son auteur, ni encore avec le devenir des choses italiennes du temps présent.

Pour réaliser un texte où se manifestent les ruptures, les allusions, les parodies, les citations, les exagérations de l'auteur, etc., il faut que des différences apparaissent, que le texte ne soit pas unifié dans une géométrie, dont la rection semblerait appartenir à celui qui l'écrit, comme cela se pratique dans la quête unifiante d'une histoire, opérée dans tant d'œuvres, et qui constitue également un double gommage: et de la textualité et du réel. En revanche, la différence doit être inscrite au cœur de l'œuvre, si on veut tenter un dépassement. Comment? Dans quelle mesure? Tel est le problème critique, ce qui signifie, pour un lecteur qui interroge et s'interroge, ne pas commencer par les universaux du méta- ou de l'anti-roman historique, ni non plus par la problématique des marginaux.

Je ne quitte pas le texte de 1997, intitulé "Il sorriso, vent'anni dopo": environ à la moitié, Consolo évoque sa découverte de Cefalù, de l'érudit ottocentesco Enrico Pirajno di Mandralisca, et surtout du Ritratto d'ignoto di Antonello da Messina, tradizionalmente detto dell'Ignoto marinaio, trovato dal Mandralisca a Lipari, nella bottega di uno speziale. Dans le paragraphe suivant, le tableau est simplement indiqué comme il Ritratto, avec une majuscule.

Donc: il Ritratto, l'Ignoto marinaio, Ritratto d'ignoto di Antonello da Messina. Et titre métonymique que Consolo utilise pour l'évocation rétrospective de son roman renvoyant au tableau: Il sorriso. Il y a des différences à exploiter.

Du livre de Consolo, la recension la meilleure a sans doute été celle de Leonardo Sciascia. Elle figure dans Cruciverba (1983), où sous le titre L'ignoto marinaio, elle précède immédiatement une nota, intitulée L'ordine delle somiglianze, consacrée à Antonello da Messina, et d'où est extraite la seconde épigraphe du Ritratto. Du texte sciascien, évidemment antérieur au roman de Consolo, je tire ceci:

A chi somiglia l'ignoto del Museo Mandralisca? Al mafioso della campagna e a quello dei quartieri alti, al deputato che siede sui banchi della destra e a quello che siede sui banchi della sinistra, al contadino e al principe del foro, somiglia a chi scrive questa nota (ci é stato detto); e certamente somiglia ad Antonello. E provatevi a stabilire la condizione sociale e la particolare umanità del personaggio. Impossibile. È un nobile 
o un plebeo? Un notaro o un contadino? Un uomo onesto o un gaglioffo? Un pittore un poeta un sicario?

"Somiglia", ecco tutto.

Ce paragraphe n'échappe pas à la contradiction consciente, énigmatique pour son auteur, qui parcourt tout le raisonnement sciascien servant de base à l'analyse de l'oeuvre d'Antonello, en relation directe avec la réalité sicilienne, laquelle se doit d'être historique, en devenir, et qui, pourtant, semble être une essence dans sa fixité. Mais ce qui se donne à regarder chez le peintre sicilien c'est, curieusement, l'essence même de la ressemblance, et ce à l'intérieur d'une société transhistorique qui, de la ressemblance justement, fait une clé de lecture de l'intersubjectivité.

Il faudrait être distrait pour ne pas apercevoir le lien, et même la dialectique, entre différence et ressemblance. Les Siciliens sont, plus que d'autres peut-être, en quête des ressemblances. Reste que, pour enregistrer une ressemblance, il faut d'abord qu'ait été établie une différence, pas de ressemblance sans différence préalable. Si bien que la ressemblance ne fait que traduire une plus juste évaluation d'un point du réel, la reconnaissance d'une limite. Nous avons affaire à une correction, la différence n'apparaissant plus comme un absolu, si bien que la ressemblance peut être dite un mode d'intériorisation de la différence.

On a soutenu que ce qui ne ressemblait à rien n'existait pas. Il n'y a que la mort, "annidata, accettata, vagheggiata», dans n'importe quelle pensée du Sicilien, observe Sciascia, pour satisfaire en toute rigueur, croyonsnous, à cette absolue condition. La mort seule ne ressemble à rien puisqu' elle est justement le rien, encore que nous ne cessions de nourrir notre imagination de ses représentations, qui en fait ne constituent que des pressentiments. En revanche, tout ce qui existe a une ressemblance possible, même s'il faut qu'un sujet l'articule. Le réel est un ensemble instable d'objets, séparés les uns des autres par leur différence ontologique, dans laquelle on discernera un maillage multidimensionnel. La ressemblance est une façon de contourner la différence en tel ou tel point, de l'accepter tout en la contestant. L'ordre des ressemblances est alors comparable à un tissage que le sujet propose pour lire la part du réel qu'il saisit.

Dans Il sorriso dell'ignoto marinaio, au chapitre premier, le noble Mandralisca transite de l'île de Lipari, où il a acquis le Ritratto dell'ignoto marinaio, vers la côte de la Sicile. Il ne quitte pas cette tavoletta, qu'il a même attachée à son vêtement, comme s'il voulait, physiquement, ne faire qu'un avec l'objet, qui est un portrait, et avec lequel il a veillé toute la nuit de la traversée. On dira encore que le baron y est attaché. Mais celui- 
ci est interpellé par un curieux personnage - «uno strano marinaio » - qui ressemble à quelqu'un qu'il connaît, se répète-t-il. Mais à qui? En vain il cherche qui peut être ce faux inconnu. Exemple de mémoire différée, le souvenir ne lui reviendra que rétrospectivement, et en deux temps. Le personnage énigmatique était Interdonato, déguisé en marin, pour mieux jouer son rôle de conspirateur. Au chapitre 2, il se donnera pour marchand. La fiction romanesque, la fiction tout court, en effet, jettent le lecteur dans les années qui précèdent la conclusion sicilienne de l'Unità.

Par jeu d'écriture intercalé, Consolo manipule le tableau d'Antonello. Il en tire la figure du conspirateur, qui avait été, tout comme Mandralisca, membre du parlement sicilien en 1848 : un lecteur avisé reconnaît, dans la description du personnage, un équivalent textuel, ou presque, du portrait quattrocentesco, en particulier dans l'insistance sur le sourire. En s'interrogeant sur l'identité de l'étrange marin, qui lui a aussi indiqué, le plus simplement du monde, que, à bord, l'homme souffrant des poumons, en quête d'une guérison miraculeuse, était un "cavatore di pietra pomice», le noble Pirajno ne vit pas une expérience différente de celle que connaît quiconque a regardé le tableau d'Antonello, et que Sciascia a saisie: le personnage du tableau ressemble, mais à qui? Et c'est précisément cela qui échappe. À la fin du premier chapitre du roman, quand le tableau est publiquement dévoilé, dans la demeure de Mandralisca, celui-ci a comme une illumination: le marin inconnu sur le bateau entre Lipari et Tindari était, étrange image, semblable au personnage de Antonello da Messina.

L'enjeu c'est le portrait. Le portrait de qui? Et qu'est-ce qu'un portrait? À l'intérieur d'un système pictural fondé sur la représentation, comme celui de ce qu'il est convenu d'appeler «l'Occident», le portrait constitue sans doute la réalisation parfaite d'un désir du peintre ou de son modèle. Non pas que le peintre fasse basculer, en le fixant sur la toile, un instant de la vie d'un être humain, exprimé par son visage, dans l'éternité. Cela ne sera même pas vrai avec la photographie. Faire un portrait, picturalement, signifie saisir d'un sujet un quid sensible, qui, en quelque sorte, est son identité, et la restituer en le reproduisant sur une surface, d'où l'illusion que ce que je vois sur le tableau coïncide avec l'image que j'ai de cette personne, ou qu'elle a d'elle-même.

Le portrait constitue un cas du rapport entre différence et ressemblance, le cas limite, vu qu'au travers de ce dessein pictural se poursuit l'abolition d'un écart, le peintre visant l'identité de deux images: celle que j'ai de la personne que je vois, et celle du tableau que je regarde. Ne dit-on pas, en forme de compliment, que la ressemblance est parfaite? Mais, dans cette formulation, l'adjectif altère le substantif, on tend à suggérer, en fait, 
l'identité, car la ressemblance, par rapport justement à l'identité, sera toujours l'aveu d'une imperfection. Ainsi pensée, elle est comme le signe d'une défaite: la différence n'a pas été abolie, malgré le désir du peintre ou de son commettant.

Il faut recourir à la mort. Le portrait poursuit la mort de celui qu'il représente. Quand le modèle meurt, nous en possédons enfin l'essence, le vivant n'étant plus là pour le dégrader en ressemblance, et contester la perfection supposée de l'identité. Avec perfidie, on ajoutera: combien d'hommes plus ou moins illustres ont, de leur vivant, cherché à ressembler à leur soi-disant portrait! Et maintenant, les voici enfin tels que l'éternité les change... Le portrait vise une identité individuelle, la ressemblance est bien le moins qu'on puisse en attendre, le peintre sait qu'il faut aller audelà.

À moins que ne se produise un accident. Le portrait devait éterniser un mortel, mais, pour quelque raison inconnue, on a perdu jusqu'au nom et à la fonction de cet homme, et la philologie est impuissante à fournir une conclusion acceptable. Objectivement, ce portrait peut être celui de quiconque, et donc le portrait de personne. Il faut se résigner: portrait d'inconnu. Pourquoi ne serait-ce pas un marin? Impossible, proclame l'historien de l'art, à l'époque, pas de tableau de genre, ça ne saurait être un marin, Roberto Longhi paraît chagrin que les littérateurs reprennent cette légende. Pourtant, comme le prouvent Sciascia et Consolo, c'est la littérature qui tient la route, car un tableau, aussi prestigieux soit-il, appartient autant aux gens de lettres qu'aux philologues de l'art. Et même, un tableau, n'importe quel tableau, appartient à quiconque: sur ce tableau je suis libre de projeter ma propre identité, de me confronter avec lui.

Dans le cas du Ritratto d'ignoto de Antonello da Messina, qui se trouve à Cefalù, où l'avait recueilli Pirajno di Mandralisca, on est en présence d'un portrait en quête d'un modèle, et tout Sicilien, à quelque époque qu'il appartienne, est candidat à ce rôle, peut être ce personnage. La capture de l'identité est inversée: le peintre entendait s'emparer d'une identité individuelle, alors que, maintenant, des individus s'interrogent, sont l'objet d'une interrogation relative à leur ressemblance avec ce portrait, qui pourtant n'est pas le leur, puisqu'il n'y a qu'une ressemblance. Et si la ressemblance valait plus que l'identité?

Sciascia, Consolo disent que tel ou tel Sicilien ressemble à ce portrait. lls sont plusieurs, un nombre non fini, à lui ressembler. C'est à partir de là qu'est construit Il sorriso dell'ignoto marinaio. Au fond, cette ouvre d'Antonello, grâce à un manque, est devenue comme l'essence du Sicilien, ou, à tout le moins, d'un certain nombre d'entre eux. Le portrait reste une 
image, cela signifierait-il que l'essence du Sicilien est imaginaire? Ou encore approchée, fausse? Joli paradoxe pour une essence, mais conforme à une certaine idée du Sicilien. Effectivement, dans le cas du conspirateur Interdonato, la ressemblance réelle avec le portrait côtoie le déguisement, l'écriture permet une sorte d'osmose, au point que, trouvaille romanesque, la fiction fournit cette désignation récusée par la philologie historique: Ignoto marinaio. Le lecteur doit attendre la fin du chapitre premier pour que Mandralisca commence à comprendre, et ce n'est qu'au chapitre 2 que le baron débrouillera la perception confuse initiale. Il écrit au chapitre 6, à Interdonato, à propos du legs qu'il entend faire de ses livres et collections:

I libri e la ricolta d'antichità e dipinti saranno una pubblica biblioteca e un museo, nel quale risplenderà come un gioiello, voi già sapete, quel ritratto d'ignoto d'Antonello, a voi sì simigliante... E forse un poco anche a me, ma pure al pittore Bevelacqua, a mio cugino Bordonaro, al vescovo di qua Ruggiero Blundo, e infino, e ciò mi duole, al già ministro borbonico Cassisi e al direttore di polizia Maniscalco... Sapete? A furia di guardarlo, quell'uomo sconosciuto, qui nel mio studio, in faccia allo scrittoio, ho capito perché la vostra fidanzata, Catena Carnevale, l'ha sfregiato, proprio sul labbro, appena steso in quel sorriso lieve, ma pungente, ironico, fiore d'intelligenza e sapienza, di ragione, ma nel contempo fiore di distacco, lontananza (come quella materiale vostra d'un tempo, per mari e porti e capitali d'Europa e d'Africa), d'aristocrazia, dovuta a nascita, a ricchezza, a cultura o al potere che viene da una carica...

Ho capito: lumaca, lumaca è anche quel sorriso!

Autour de ce passage, d'autres se regroupent, chaque fois que le mot sorriso, ou le verbe sorridere, ou riso-ridere, ou encore quelque terme qui semble renvoyer au personnage d'Antonello, apparaissent dans le texte, le lecteur se fait vigilant, pour capter les fragments du tableau irradiant. J'interprète rétrospectivement l'aveuglement du premier chapitre: c'est, paradoxalement, la ressemblance qui trompe Mandralisca, en même temps que son désir inconscient de collectionneur, d'être lui seul semblable au personnage de ce tableau. Je ne néglige pas la lecture qu'Interdonato proposait du geste de la fille du pharmacien Carnevale, au chapitre 2 intitulé L'albero delle quattro arance, ainsi que ces autres portraits, de l'Italie cette fois, que sont la Koré grecque et la broderie faussement confuse en forme d'arbre à l'envers. Ce thème du portrait, jeu stylistique de la ressemblance, plutôt que de l'identité, très fort dans les deux premiers chapitres, va se réduire par la suite, sans jamais disparaître pour autant. Les objets en présence et la structure même des deux premiers chapitres du roman sont à mettre en parallèle, car ils se ressemblent et s'opposent. Se ressembler et s'opposer, telle est aussi la structure du château du 
chapitre 8, Il carcere. Mais dans la catastrophe d'Alcàra Li Fusi, je ne vois plus le Ritratto dell'ignoto marinaio. Toutefois, il est repérable encore, au début du chapitre 7, de façon ambiguë, quand Mandralisca se souvient qu'il a transcrit les poèmes des prisonniers: «il riso dell'Ignoto, a me davanti, al tremolìo del lume, da lieve e ironico mi parve si volgesse in greve, sardonico, maligno... ». Je n'oublie pas le plan de Cefalù peint par Bevelacqua, que le baron tient dans son bureau près du portrait d'Antonello, car c'est une façon de pourtraire la ville. L'écriture consolienne enfin est portrait du langage.

Le thème dominant dans toute l'œuvre de Vincenzo Consolo est la catastrophe: historique, psychique, linguistique, cosmique... mathématique peut-être. Du moins est-ce là un horizon de lecture. Il sorriso dell'ignoto marinaio (1976) renferme une catastrophe au sens courant de ce terme: la révolte de Alcàra Li Fusi. L'Unità, pour la Sicile, a été une catastrophe: oui et non, au sens du Verga de Viva la libertà, oui et non au sens de quelque noble pirandellien de I vecchi e i giovani. Mais Consolo a aussi décrit la catastrophe en tant que telle, et non pas seulement comme jugement idéologique ou comme vécu de classe. Quand il montre Alcàra Li Fusi après la révolte et sa répression, ce qu'il dit c'est la catastrophe, le résultat. Le lecteur n'assiste pas à la jacquerie, le personnage qui raconte est arrivé, dirons-nous, après la bataille, il constate. Le recours à la métonymie systématique, au sens de Jakobson, conduit, dans le récit, à une représentation disloquée de ce qui tombe sous les sens, il n'y a plus d'ordre structuré des choses, d'ordre des ressemblances, tandis que l'emploi d'expressions de la liturgie enracine le texte dans un contexte chrétien, de fin du monde En position dominante, reparaît le moine épileptique, c'està-dire atteint du mal caduco, personnage central du chapitre III intitulé Morti sacrata. L'épisode de la révolte lui-même s'est passé dans le Val Demone, lieu dont le signifiant évoque le diable...

La catastrophe - il suffit de songer au théâtre - ne se situe pas dans un avant et un après, c'est un acte final, dernier et principal événement d'un poème, d'une tragédie, mais aussi d'un roman, d'une séquence historique. Elle est à peine moins définitive que la mort, uniformisante, elle abolit tout, et jusqu'à la différence. La mort, précisément, en est souvent une composante. Dans le roman de Consolo, la catastrophe inscrite dans l'histoire (mais en Sicile, on ne connaît de fin de l'histoire qu'en apparence, ça ne cesse de continuer) en induit une autre qui s'inscrit dans la vie d'un individu, puisque le personnage principal, Pirajno di Mandralisca, se convertit à la suite de cet événement. Il s'agit bien d'une conversion, car le baron passe d'une croyance qui lui apparaît comme fausse à la vérité. Il 
change ontologiquement. Il cesse de croire en la différence qui fondait la noblesse, pour penser un horizon humain sans classe. Le lecteur du Sorriso dell'ignoto marinaio estimera peut-être que la fin des temps apocalyptique ou bien, dans une version laïque, la fin de l'histoire, c'est toujours ce même concept d'une catastrophe finale qui hante l'humanité et constitue l'arrière-plan de toutes les catastrophes locales traversant la vie de chacun et l'histoire des sociétés.

Non pas identifiable aux faits de Alcàra Li Fusi, mais en fonction de ceux-ci, un élément différentiel devient décisif idéologiquement, narrativement, structurellement, dans le personnage de Mandralisca, qui cesse d'être un noble érudit et savant, accumulant de la connaissance en soi (emblématiquement, tout savoir sur les escargots siciliens...), pour devenir le défenseur des révoltés du Val Demone auprès d'Interdonato, un personnage important dans le nouveau régime, qui n'a plus besoin de se déguiser en marin, mais à Messine est devenu procureur.

Dans la fiction consolienne, comme il se doit, la conversion du baron ne s'identifie pas à une illumination subite et sans antécédent. Des faits qui sont l'histoire ont happé ce collectionneur, ce docte, qui avait siégé au Parlement de Sicile en 1848, qui avait hébergé des conspirateurs en 1856, qui avait été torturé pour cette raison, qui savait d'avant encore ce qu'était la respiration d'un homme rongé par la silicose, qui avait interpellé et été interpellé ensuite par un prisonnier de Maniforti, qui avait vu Alcàra Li Fusi après la jacquerie... si bien qu'il finit par parler comme les grands socialistes de son siècle. Ce qui fait de ce personnage le héros d'une œuvre, quand bien même celle-ci joue sur la discontinuité, c'est, comme dans tout roman, la signification exemplaire que le lecteur perçoit: dans $I l$ sorriso dell'ignoto marinaio, le cheminement intérieur et public de Pirajno di Mandralisca s'identifie à la crise de sa propre identité nobiliaire, il plaide pour les hommes de Alcàra Li Fusi, parce qu'il ne s'éprouve plus comme noble, mais comme homme.

Un noble est un être différent. Il peut choisir sa différence, en étant érudit, collectionneur, et même le plus grand expert de «malacologia terrestre e fluviatile di Sicilia». Mais ce qui fait la noblesse, c'est, fondamentalement, une différence collective, de fait une appartenance: être noble, c'est ne pas être comme tous les autres. Comment saisir le fondement de cette proposition et son extension? Tel est le questionnement qui se développe au travers du personnage, qui entre en crise vraiment, c'est-à-dire dont l'identité, à ses propres yeux, ne va plus avoir de fondement. Le point de départ est vittorinien, car il s'agit toujours de la même question: Uomini e no? Le noble appartient à un groupe, à une classe, encore que 
réduite en nombre; entre ceux qui la composent règne une sorte d'égalité, et, malgré les différences individuelles, une complicité de fait. Les autres, qui ne sont pas nobles, peuvent être traités comme s'ils n'étaient pas des hommes, car la sélection nobiliaire engraine un mécanisme d'exclusion. Cela n'est pas vrai pour les serviteurs et quelques autres qui sont comme absorbés par le monde personnel du maitre et des maitres, qui y participent et en tirent profit. Il en va autrement des paysans, qui constituent la classe antagoniste. Que dire des révoltés, des captifs, qui sont les vrais marginaux?

L'ultime strate du réel est la richesse, à savoir, à la fin du dix-neuvième siècle en Sicile, comme dans beaucoup d'autres contrées du globe, la terre. Les nobles la possèdent, les paysans la travaillent. Il existe aussi d'autres possédants avec lesquels faire bloc. L'avoir engendre une différence ontologique entre les hommes, que le socialisme, utopique ou non, entendait briser, le recours à la violence ne pouvant être tu. D'où la position incarnée par Mandralisca, par Consolo et d'autres: le Risorgimento en Sicile ne saurait se soustraire à une interrogation sur la violence. L'Italie, oui, mais quelle société?

La spécificité du roman historique, par rapport à une recherche dite objective, tient à ce qu'il permet, à travers la subjectivité de l'écrivain, une réflexion critique vivante. Impossible de s'abriter derrière ce qu'on nomme les faits, et qui n'est le plus souvent qu'une apologie justificatrice de ceux qui, dans une certaine conjoncture, l'ont emporté. Le Mandralisca consolien est moins situé dans l'histoire qu'il ne montre la crise de la revendication nobiliaire en tant que justification idéologique typique de n'importe quelle classe dominante. La noblesse a pu compter davantage en Sicile qu'ailleurs, et toute justification est une fable, soutient justement le romancier.

On ne se débarrasse pas facilement de l'identité nobiliaire pour autant, on aimerait qu'il en restât, sur la base d'un meilleur fondement, la marque distinctive, que quelque chose continue à lui ressembler. Mandralisca est hanté par la question, il a de la sympathie pour ce que nous nommerions méritocratie, il voit aussi en Interdonato un homme noble... Mais il comprend la position de Catena, qui est la fiancée d'Interdonato: être noble, c'est faire le choix d'une attitude, celle-là même qu'a saisie Antonello dans ce portrait ${ }^{2}$, dans ce sourire notamment. Il faut donc aboutir au rejet de ce sourire que la peinture a fixé, mais qui est d'abord le mouvement d'un visage réel. Le livre de Consolo ne dépend pas particulièrement d'une thématique idéologique que les dernières années $60 \mathrm{du} \mathrm{XX}^{\mathrm{e}}$ siècle auraient favorisée. Il donne à voir des différences, un culte trompeur de la diffé- 
rence, que la répartition, ou plus justement la captation de la richesse engendre, et qui conduit à une tragédie, à la catastrophe, face à laquelle il ne reste plus, pour le juste, qu'à tenter, après coup, de faire en sorte qu'elle ne serve pas, dans une logique de la culpabilité, à garantir la vengeance.

Pirajno voudrait renverser la dialectique de la différence. Comme baron, il est différent, parce que, en dernière analyse, la possession de la terre n'est possible que du fait qu'il y a des paysans qui n'ont rien et qui travaillent ses terres (i feudi). Il ne peut pas s'identifier à eux, et ne saurait parler en leur nom. Réciproquement, le discours des paysans ne peut être qu'autre, fondé sur un autre code à trouver, pour exprimer, en particulier, leur comportement et sa justification à l'égard de la violence. Une société où les paysans sont des sujets, articulent eux-mêmes leur dire, c'est une société différente, où le rapport à la propriété aurait changé. Ce dessein n'est-il que la dernière illusion d'un noble idéaliste, dont on se gausserait, s'il ne fallait en sourire? Pour le moment, au lendemain de l'Unità, il reste à faire (morale provisoire?) quelque chose pour sauver la vie des paysans qui ont laissé exploser leur violence, dans la mesure où un noble éclairé en saisit la logique, enracinée dans une société qui, elle, doit être changée. De là cette plaidoirie adressée à Interdonato, qui semble avoir porté ses fruits, à en juger par le premier appendice au chapitre 9, qui constitue également une variation stylistique et éthique sur la différence.

Je ne crois pas que Il sorriso dell'ignoto marinaio soit un roman ésotérique, ou qu'il dise tout sur la condition humaine au-delà de ce que cherche toujours à exprimer un écrivain dans une ouvre fondatrice. $\mathrm{La}$ spirale, la cycloïde, la coquille d'escargot... n'y est pas la clé du monde ou de l'histoire, je vois manifestée, par cette forme, la cohérence d'un personnage, vraisemblablement selon une approche matérialiste: il se convertit, mais sa pensée est analogique d'un objet constant. Par ailleurs, la spirale appartient à la culture musulmane, comme la Koré à la Grèce; l'une et l'autre appartiennent à la Sicile et l'ont faite, comme l'Espagne, comme l'Italie, la réciproque étant également vraie, si bien que la Sicile est aussi la Grèce, les arabes, les chrétiens, l'Hispanidad, l'Italie... Elle est ce palimpseste de différences superposées, qui fait que son roman historique, dans la deuxième moitié $\mathrm{du} \mathrm{XX}^{\mathrm{e}}$ siècle, ne peut pas être univoque, linéaire, mais, comme Il sorriso dell'ignoto marinaio, charrie une matière sédimentée, éparse apparemment.

L'écriture consolienne, dans son baroquisme, son refus de l'uniformité dérivée des Lumières, non pas du fait d'une position réactionnaire, mais au contraire pour que le dire soit réel autant que combatif, exprime une diversité, le refus de l'uniformisation et de l'homologation. Planté au 
cœur du langage, de l'historiographie, qu'il évoque et qu'il suppose connue de son lecteur, l'écrivain imagine, en relation avec la catastrophe, deux écritures: celle du baron et celle des prisonniers que la répression a frappés. Au long du roman, les différences d'écritures ont été multiples, selon la source rédactionnelle (archives, citations ou pseudo-citations...), selon les épisodes et les personnages romanesques (variations et variantes de styles narratifs), mais c'est dans l'opposition qui, bien sûr, n'est pas que formelle, entre la description objective de la catastrophe, et ces sortes de poèmes où les révoltés disent et se disent à la première personne, que la différence prend pleinement son sens, qu'elle devient la raison d'être d'un paradoxal plaidoyer. L'écrivain n'est pas hors de la société, mais au travers de la différence des écritures, il se doit d'imaginer. Il n'est ni le baron ni le paysan en révolte: il fait percevoir la différence en même temps qu'un référent commun tragique.

L'ordre des ressemblances reste présent à la conscience de celui qui écrit, et de celui qui lit ou relit Il sorriso dell'ignoto marinaio. Consolo a vu la fin, en Sicile, de la société que ses personnages sont censés incarner et représenter, mais il a connu également Milan, l'Italie... dans les années 60-70. Aujourd'hui je regarde la planète unifiée par le libéralisme, et je perçois des ressemblances avec ce que me donnait à imaginer le romancier, il y a plus d'un quart de siècle. Si Consolo était un idéologue, il n'aurait pas écrit ce livre, mais quelque traité, quelque essai. Or il a écrit un roman, et on pensera que des figures réelles de noble sicilien, d'illuminista et d'illuminé, de révolté, de femme... l'ont interpellé, ont nourri sa méditation, et l'ont conduit à écrire un chef d'œuvre, où des personnages leur ressemblent.

\section{Notes}

1. Ma bibliographie se limite au numéro de Nuove effemeridi, Anno VIII, n. 29, 1995/1 et à l'essai de G. Traina, Vincenzo Consolo, Cadmo, 2001.

2. N'étant ni sicilien ni noble, je me situerai par rapport à cet auto-portrait sartrien que sont Les mots: «Si je range l'impossible Salut au magazin des accessoires, que reste-t-il? Tout un homme, fait de tous les hommes et qui les vaut tous et que vaut n'importe qui». 